\title{
SpringerBriefs in Mathematics
}

\author{
Series Editors \\ Krishnaswami Alladi \\ Nicola Bellomo \\ Michele Benzi \\ Palle E.T. Jorgensen \\ Tatsien Li \\ Roderick Melnik \\ Matthias Neufang \\ Otmar Scherzer \\ Dierk Schleicher \\ Benjamin Steinberg \\ Lothar Reichel \\ Yuri Tschinkel \\ Loring W. Tu \\ G. George Yin \\ Ping Zhang
}

SpringerBriefs in Mathematics showcases expositions in all areas of mathematics and applied mathematics. Manuscripts presenting new results or a single new result in a classical field, new field, or an emerging topic, applications, or bridges between new results and already published works, are encouraged. The series is intended for mathematicians and applied mathematicians.

More information about this series at http://www.springer.com/series/10030 

Michael Grabchak

\section{Tempered Stable Distributions}

Stochastic Models for Multiscale Processes 


\author{
Michael Grabchak \\ Department of Mathematics and Statistics \\ University of North Carolina \\ Charlotte, NC, USA
}

ISSN 2191-8198

SpringerBriefs in Mathematics

ISBN 978-3-319-24925-4

DOI 10.1007/978-3-319-24927-8
ISSN 2191-8201 (electronic)

ISBN 978-3-319-24927-8 (eBook)

Library of Congress Control Number: 2015957382

Mathematics Subject Classification (2010): 60E07, 60G51, 60G52

Springer Cham Heidelberg New York Dordrecht London

(C) Michael Grabchak 2016

This work is subject to copyright. All rights are reserved by the Publisher, whether the whole or part of the material is concerned, specifically the rights of translation, reprinting, reuse of illustrations, recitation, broadcasting, reproduction on microfilms or in any other physical way, and transmission or information storage and retrieval, electronic adaptation, computer software, or by similar or dissimilar methodology now known or hereafter developed.

The use of general descriptive names, registered names, trademarks, service marks, etc. in this publication does not imply, even in the absence of a specific statement, that such names are exempt from the relevant protective laws and regulations and therefore free for general use.

The publisher, the authors and the editors are safe to assume that the advice and information in this book are believed to be true and accurate at the date of publication. Neither the publisher nor the authors or the editors give a warranty, express or implied, with respect to the material contained herein or for any errors or omissions that may have been made.

Printed on acid-free paper

Springer International Publishing AG Switzerland is part of Springer Science+Business Media (www. springer.com) 
To Lijuan 



\section{Acknowledgments}

I would like to thank Professor Gennady Samorodnitsky, my PhD advisor, who first introduced me to tempered stable distributions and spent many hours discussing them with me. I likewise thank Donna Chernyk, editor at Springer, who first suggested the possibility of me writing this brief and then helped me through the entire process. I also thank the many anonymous referees who read the manuscript both while it was being written and after the first draft was completed. Their valuable comments and suggestions led to improvements in the presentation of this brief. Finally, I thank my family for their support and patience. 



\section{Contents}

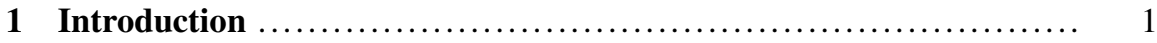

2 Preliminaries ................................................. 5

$2.1 \quad$ Basic Topology ........................................ 5

2.2 Infinitely Divisible Distributions and Lévy Processes............... 6

2.3 Regular Variation........................................ 9

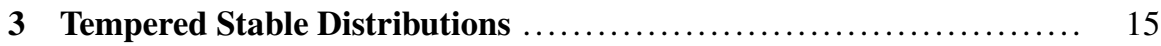

3.1 Definitions and Basic Properties .......................... 15

3.2 Identifiability and Subclasses............................... 24

3.3 Tails of Tempered Stable Distributions ........................ 31

3.4 Tempered Stable Lévy Processes ............................. 34

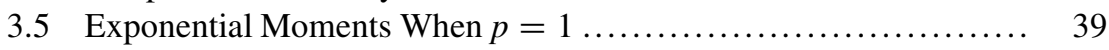

4 Limit Theorems for Tempered Stable Distributions .................. 47

$4.1 \quad$ Extended Tempered Stable Distributions ...................... 47

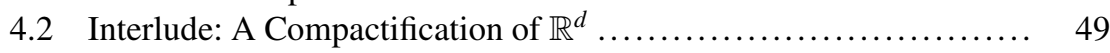

4.2.1 Definitions ...................................... 49

4.2.2 Vague Convergence ............................... 50

4.3 Extended Rosiński Measures ................................. 55

4.4 Sequences of Extended Tempered Stable Distributions ............ 57

4.5 Closure Properties......................................... 63

5 Multiscale Properties of Tempered Stable Lévy Processes ............ 67

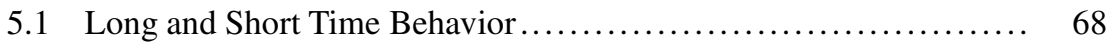

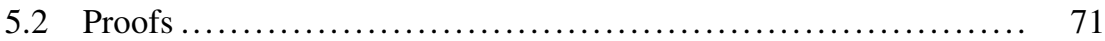

6 Parametric Classes ........................................... 83

6.1 Smoothly Truncated Lévy Flights ......................... 83

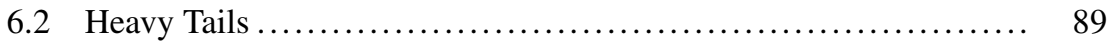

6.2.1 Power Tempering ................................... 89

6.2.2 Gamma Tempering $\ldots \ldots \ldots \ldots \ldots \ldots \ldots \ldots \ldots \ldots \ldots \ldots \ldots \ldots \ldots \ldots \ldots \ldots \ldots$

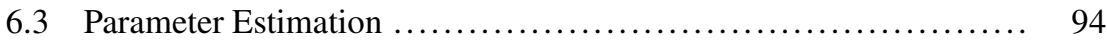




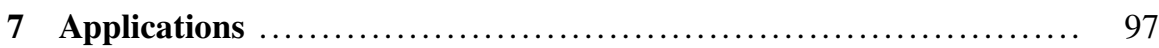

7.1 Option Pricing ............................................. 97

7.2 Mobility Models .................................... 102

7.3 How Do Tempered Stable Distributions Appear in Applications?... 104

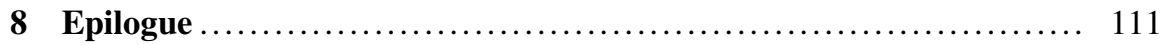

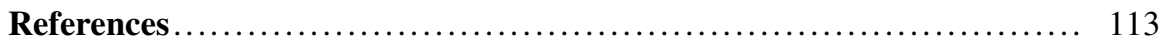

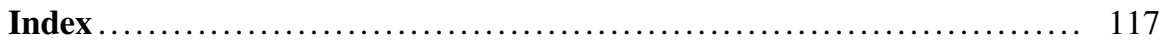




\section{Notation}

Let $\mathbb{R}^{d}$ be the space of $d$-dimensional column vectors of real numbers. For $x \in \mathbb{R}^{d}$ we write $x=\left(x_{1}, x_{2}, \ldots, x_{d}\right)$, and we denote the transpose of $x$ by $x^{T}$. Let $\langle\cdot, \cdot\rangle$ be the usual inner product on $\mathbb{R}^{d}$, i.e., if $x, y \in \mathbb{R}^{d}$ then $\langle x, y\rangle=x^{T} y=\sum_{i=1}^{d} x_{i} y_{i}$. Let $|\cdot|$ be the usual norm on $\mathbb{R}^{d}$, i.e., if $x \in \mathbb{R}^{d}$ then $|x|=\sqrt{\langle x, x\rangle}=\sqrt{\sum_{i=1}^{d} x_{i}^{2}}$. Let $\mathbb{S}^{d-1}=\left\{x \in \mathbb{R}^{d}:|x|=1\right\}$, and let $\mathbb{C}^{d}$ be the space of $d$-dimensional column vectors of complex numbers. For $z \in \mathbb{C}^{d}$ we write $\Re z$ to denote the real part of $z$ and $\mathfrak{s} z$ to denote the imaginary part of $z$. Let $\mathbb{R}^{d \times d}$ be the collection of all $d \times d$-dimensional matrices with real entries, and for $A \in \mathbb{R}^{d \times d}$ let $\operatorname{tr} A$ be the trace of $A$. Let $\mathfrak{B}\left(\mathbb{R}^{d}\right)$ and $\mathfrak{B}\left(\mathbb{S}^{d-1}\right)$ denote the Borel sets on $\mathbb{R}^{d}$ and $\mathbb{S}^{d-1}$, respectively.

If $\mu$ is a probability measure on $\left(\mathbb{R}^{d}, \mathfrak{B}\left(\mathbb{R}^{d}\right)\right)$, we write $\hat{\mu}(z)=\int_{\mathbb{R}^{d}} e^{i\langle x, z\rangle} \mu(\mathrm{d} x)$ for $z \in \mathbb{R}^{d}$ to denote its characteristic function, and we write $X \sim \mu$ to denote that $X$ is a random variable taking values in $\mathbb{R}^{d}$ with distribution $\mu$. Further, we write $X_{1}, X_{2}, \ldots, X_{n} \stackrel{\text { iid }}{\sim} \mu$ to denote that $X_{1}, X_{2}, \ldots, X_{n}$ are independent and identically distributed random variables taking values in $\mathbb{R}^{d}$, each with distribution $\mu$. For two random variables $X$ and $Y$, we write $X \stackrel{d}{=} Y$ to denote that $X$ and $Y$ have the same distribution.

For a sequence of random variables $X_{1}, X_{2}, \ldots$, we write d-lim $X_{n}$ to denote the limit in distribution. We also use the more standard notations $\stackrel{d}{\rightarrow}$ and $\stackrel{p}{\rightarrow}$ to denote, respectively, convergence in distribution and convergence in probability. For sequences of probability measures, we write $\stackrel{w}{\rightarrow}$ to denote weak convergence, and for sequences of Radon measures, we write $\stackrel{v}{\rightarrow}$ to denote vague convergence.

We write $I D(A, M, b)$ to denote the infinitely divisible distribution on $\mathbb{R}^{d}$ with Gaussian part $A$, Lévy measure $M$, and shift $b$. We write $N(b, A)$ to denote the Gaussian distribution on $\mathbb{R}^{d}$ with mean vector $b$ and covariance matrix $A$, and we write $S_{\alpha}(\sigma, b)$ to denote the infinite variance $\alpha$-stable distribution on $\mathbb{R}^{d}$ with spectral measure $\sigma$ and shift $b$. We write $T S_{\alpha}^{p}(R, b)$ to denote the $p$-tempered $\alpha$-stable distribution on $\mathbb{R}^{d}$ with Rosiński measure $R$ and shift $b$, and we write $\operatorname{ETS}_{\alpha}^{p}(A, v, b)$ to denote the extended $p$-tempered $\alpha$-stable distribution on $\mathbb{R}^{d}$ with Gaussian part $A$, extended Rosiński measure $v$, and shift $b$. Several parametric families of 
tempered stable distributions are introduced in Chapter 6. These are denoted by $S T L F_{\alpha}^{p}\left(c_{-}, c_{+}, \ell_{-}, \ell_{+}, b\right), T W_{\alpha}^{p}(c, \ell, b), T W_{\alpha}(c, \ell, b), P T_{\alpha}^{p}\left(c_{-}, c_{+}, \nu_{-}, v_{+}, b\right)$, and $G T_{\alpha}^{p}\left(c_{-}, c_{+}, v_{-}, v_{+}, \ell_{-}, \ell_{+}, b\right)$.

If $\mathscr{A}$ is a collection of subsets of some space $\mathbb{E}$, we write $\sigma(\mathscr{A})$ to denote the $\sigma$-algebra generated by $\mathscr{A}$, i.e., the smallest $\sigma$-algebra that contains $\mathscr{A}$. If $f$ and $g$ are real-valued functions, $c \in\{0, \infty\}$, and $k \in \mathbb{R}$, we write

$$
f(t) \sim k g(t) \text { as } t \rightarrow c
$$

to denote

$$
f(t) / g(t) \rightarrow k \text { as } t \rightarrow c .
$$

Note that this is somewhat nonstandard notation in the case when $k=0$. When dealing with infinity we adopt the conventions that

$$
1 / \infty=0 \text { and } 1 / 0=\infty .
$$

For $x, y \in \mathbb{R}$ we write $x \wedge y$ to denote the minimum and $x \vee y$ to denote the maximum. Further, we define $\log ^{+} x:=\log (1 \vee x)$. For a set $H$ we denote the indicator function on $H$ by $1_{H}$. This means that $1_{H}(x)=1$ if $x \in H$ and $1_{H}(x)=0$ if $x \notin H$. We also write $1_{x \in H}$ to denote this. For $a \in \mathbb{R}^{d}$ we write $\delta_{a}$ to denote the Dirac delta measure at $a$. This means that for any Borel set $H$, we have $\delta_{a}(H)=1_{H}(a)$. We denote the gamma function by $\Gamma(x)$. When $x>0$ we have

$$
\Gamma(x)=\int_{0}^{\infty} e^{-t} t^{x-1} \mathrm{~d} t .
$$

Further, we extend the gamma function to any $x \in \mathbb{R}$ with $x \neq 0,-1,-2, \ldots$ by the relation

$$
\Gamma(x+1)=x \Gamma(x) .
$$

If $P$ is a probability measure on the measurable space $(\Omega, \mathscr{F})$, we write $\mathrm{E}_{P}$ to denote the expectation with respect to $P$. Further, when there is no chance of ambiguity, we write $\mathrm{E}$ instead of $\mathrm{E}_{P}$. 